\title{
Voltage Margin Control and Optimizing MTDC Grid Controllers Using Honey Bee Mating Optimization (HBMO) Algorithm
}

\author{
Ilma Haghani ${ }^{1}$, Mehran Chahkandi ${ }^{2}$, Hossein Ahmadi Kakhki ${ }^{3}$, Elahe Faghihnia ${ }^{4}$ \\ and Mahdi Zarif ${ }^{\text {5* }}$ \\ 1,2,3,4,5 Department of Electrical Engineering, Mashhad Branch, Islamic Azad \\ University, Mashhad, Iran \\ *zarif.mahdi.ir@ieee.org
}

\begin{abstract}
Recently, because of wide utilization of renewable resources, in multi terminal direct current (MTDC) grids are considered. MTDC grids control is very important. Vector control method has been taken as the method of controlling MTDC grid, which includes inner current controllers (ICC) and outer controllers. Also, in this controlling method, outer controllers are composed of active power controllers and DC voltage as active channel and reactive power controllers and AC voltage as reactive channel. Voltage margin control method is used to control DC voltage. Also MTDC grids' controllers are optimized by using Honey-bee Mating Optimization Algorithm (HMBO) and the results are compared with classic regulation mode. In this paper, a DC four-terminal test grid has been used for evaluating performance of HBMO algorithm.
\end{abstract}

Keywords: Voltage Margin Control, MTDC grid Controller, Honey Bee Mating Optimization

\section{Introduction}

Today, the need to electric energy is increasing over time, as it is a key element for modern societies, and in developing countries, is considered as one of the most important tools for promoting welfare and progress [1]. Now countries try to use renewable energies, such as wind energy, solar photovoltaic, wave and tidal energy and biofuels as electrical sources [2]. One of the most usable renewable energy sources is wind energy [2]. As there are limited appropriate places for construction of wind farms onshore, now countries are installing offshore wind farms, in which there is more space and wind speed is more, and also there is no obstacle in the open sea for installing them.

MTDC networks have high potential for connecting sea wind farms to onshore AC grids [3]. MTDC networks, while transferring AC power over long distances, eliminate large capacitive currents, therefore they are suitable for integrating wind farms in onshore AC grids [4]. DC grids, based on the nominal voltage level, are divided into three categories of low voltage $\left(\mathrm{V}_{\mathrm{DC}}<1500 \mathrm{~V}\right)$, medium voltage $\left(1500 \mathrm{~V}<\mathrm{V}_{\mathrm{DC}}<30 \mathrm{kV}\right)$ and high voltage $\mathrm{DC}\left(\mathrm{V}_{\mathrm{DC}}>30 \mathrm{kV}\right)[5]$.

Recently, due to technological advances in reliable electrical equipment, HVDC transmission systems are used more than the other two categories. HVDC transmission application can be divided into two major categories: transmission of power volume over long distances and asynchronous communications. Using HVDC transmission systems makes connection between asynchronous grids more economic and makes system performance more confident [6,7]. MT-HVDC grids are divided into three categories technologically: Current-Source Converter (CSC-HVDC), Voltage-Source Converter

Received (March 25, 2017), Review Result (December 5, 2017), Accepted (January 20, 2018)

* Corresponding Author 
(VSC- HVDC), and hybrid [8-10]. Given that VSCs have more desirable features such as full control over DC grids and ease of connecting other converters to the same DC grid, it is considered as the first and the best idea for constructing MTDC networks [11]. VSCHVDC control methods are; direct control, decoupled vector control, and power synchronization control; in which the most popular control method, is vector control, which is composed of an ICC and outer controllers, and allows full decouple control of AC and DC quantities desirably through converting AC quantities into d-q reference frame [12].

In vector control method, outer controllers include active and reactive channel that active channel is composed of active power controllers and DC voltage, and reactive channel is also composed of reactive power controllers and AC voltage [12]. In MTDC grids, different methods are used to control DC voltage, including voltage droop control method, ratio control, priority control, and voltage margin control [13]. According to the features of voltage margin control method, which will be described in following sections, in this paper, this method has been used to control DC voltage. Each of MTDC grid controllers in vector control method, uses proportional integral controllers (PI) because of strong performance of this type of controllers [14]. In order to achieve a desirable performance, a control is needed that PI controllers' parameters regulated in vector control method optimally. For optimal regulation of these controllers according to VSC features, it's better to use intelligent optimization methods instead of classic methods [15].

Yet, among intelligent algorithms used for optimizing MTDC grids controllers, we can refer to Genetic Algorithm (GA) and Particle Swarm Optimization (PSO) algorithm [16, 17]. In this article, we try to use HBMO algorithm for optimizing parameters of vector control method controllers. HBMO algorithm is a new heuristic algorithm, in which optimization process originates from real bees' behaviour in breeding. This algorithm has shown acceptable results in solving different mathematic problems in comparison with other optimization algorithms including GA [18]. Considering that till now voltage margin control method optimization has not been used, in this paper, as a new job, optimizing this control method is done, to show high potential of HBMO algorithm comparing with classic regulation of this controller. Moreover, in order to validate desirable performance of HBMO algorithm, the obtained results were applied in a four-terminal DC grid and performance of optimal controllers in a MTDC grid is observed.

The rest of this paper is as following: section 2 describes control structure of MTDC grid. HBMO algorithm is stated in Section 3. Regulation of MTDC grid controllers is also presented in Section 4. In Section 5, the results of simulation are observed. And finally in Section 6, research findings and recommendations for future works are presented.

\section{Control of MTDC Grid}

Each MTDC grid is composed of several onshore and offshore converters. Since in this research, VSC topology has been used, in order to control MTDC grids, it is enough that one VSC station be controlled. As mentioned, there are several methods for VSC control that here, since vector control method is a perfect and useful method, it is used for controlling VSC stations. By the aid of this method, we can control active and reactive powers easily and separately. Also, regulation AC and DC voltages is performed easily in this method.

In Figure 1, general structure of vector control method for a VSC station is shown. In this method, AC currents and converter voltages in point of common coupling (PCC) are converted into d-q reference frame, which are synchronized with AC grid voltage by a phase locked loop (PLL).

As it is shown in Figure 1, vector control method is composed of a series of outer controllers and an inner controller. Outer controllers themselves include active and reactive channel controllers that create reference currents for ICC. Also, ICC that is 
composed of PI regulators, like outer controllers, determines VSC reference voltage in d-q frame [13].

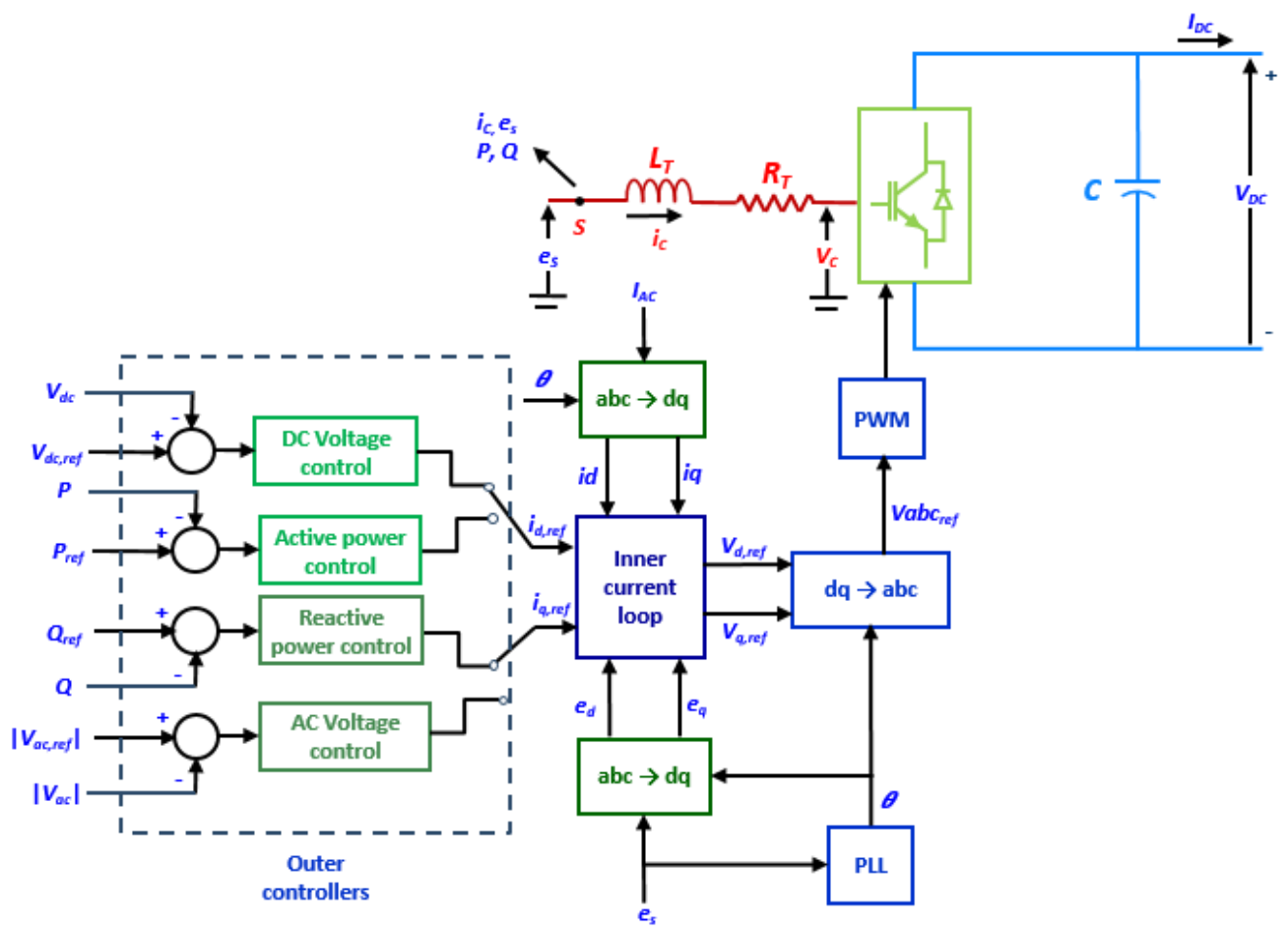

Figure 1. General Structure of $d-q$ Vector Control for a VSC-HVDC Station [13]

\subsection{Inner Current Controller (ICC)}

In VSC-HVDC vector control method, there is an inner current controller that evaluates voltage droop in reactance series $\left(X_{T}\right)$ to generate required AC current, without going beyond maximum current of converter. ICC includes fast PI controllers that follow reference currents regulated by outer controllers and generate reference voltage for the converter. Considering the structure of ICC, voltage in PCC $\left(e_{s}\right)$ and converter's voltage $\left(v_{s}\right)$ are connected by the following [13]:

$$
e_{s}-v_{s}=R_{T} i_{c}+L_{T} \frac{d i_{c}}{d t}
$$

(1) Can be rewritten as (2), in the d-q reference frame through conducting park transformation:

$$
\left\{\begin{array}{l}
e_{d}-v_{d}=R_{T} i_{d}+L_{T} \frac{d i_{d}}{d t}-\omega L_{T} i_{q} \\
e_{q}-v_{q}=R_{T} i_{q}+L_{T} \frac{d i_{q}}{d t}+\omega L_{T} i_{d}
\end{array}\right.
$$

Where RT and LT represent total resistance and inductance between converter and PCC with AC grid, and $\omega$ is also angular frequency of AC voltage in PCC.

\subsection{Outer Controllers}

As it is shown in Figure 1, outer controllers are composed of active and reactive channels that active channel is responsible of regulation active power or DC voltage, while reactive channel controls reactive power or AC voltage amplitude in PCC. Power equations are expressed in d-q reference frame as the following [13]: 


$$
P=v_{d} i_{d}+v_{q} i_{q}
$$

$$
Q=v_{q} i_{d}-v_{d} i_{q}
$$

If it is assumed that $\mathrm{d}$-axis is adapted to AC grid voltage phasor, by PLL; i.e., $v_{q}=$ 0 , then (5) and (6) obtain:

$$
\begin{gathered}
P=v_{d} i_{d} \\
Q=-v_{d} i_{q}
\end{gathered}
$$

The above relations represent this point that an independent control of active and reactive power through converter currents is possible. AC voltage control is done by changing q-axis current, so that AC voltage in PCC, follows given reference amount. The aim of DC voltage controller is maintaining DC voltage at the reference amount, by regulation converted active power with $\mathrm{AC}$ grid by regulation d-axis reference current.

\subsection{Voltage Control}

DC voltage control has a key role in reliable performance of MTDC grids. If DC voltage increase too much, protection system will be activated and high voltage droop also causes creation of non-linear phenomenon that creates problems for control system [19]. In addition, appropriate strategy of DC voltage control will ens ure power balance in grid [20].

As mentioned, different methods are used to control DC voltage in MTDC grids, including voltage droop control method, ratio control, priority control, and voltage margin method. In Table 1, a comparison between DC voltage control methods is performed in terms of dynamic response, expandability, flexibility, and communication needs. Dynamic response represents the speed of control method in minimizing transient response of DC voltage in MTDC grid during power changes in wind farms. Also, expandability indicates the possibility of DC voltage control in MTDC grids in exchange for number of nodes, while shows flexibility of each control method capability for conducting power sharing plans. Communication needs also refers to the need of each control method to input external data to implement power sharing schemes [21].

Table 1. Comparison between Different Methods of DC Voltage Control [21]

\begin{tabular}{lllll}
\hline $\begin{array}{l}\text { Control } \\
\text { method }\end{array}$ & $\begin{array}{l}\text { Dynamic } \\
\text { response }\end{array}$ & Expandability & Flexibility & $\begin{array}{l}\text { Communication } \\
\text { needs }\end{array}$ \\
\hline $\begin{array}{l}\text { Droop } \\
\text { Ratio }\end{array}$ & high & medium & low & low \\
Priority & medium & medium & medium & low \\
$\begin{array}{l}\text { Voltage } \\
\text { margin }\end{array}$ & low & high & high & medium \\
\hline
\end{tabular}

It should be noted that main feature of DC voltage control methods is in their capabilities to implement different plans of power sharing [13]. In MTDC grids, three power sharing schemes include: fixed, proportional, and priority [13]. In Table 2, a comparison between DC voltage control methods is presented in terms of implementing different schemes of power sharing. 


\section{Table 2. Different of DC Voltage Control Methods in Terms of the Capability of Implementing Power Sharing Schemes [13]}

\begin{tabular}{cccc}
\hline $\begin{array}{c}\text { Control } \\
\text { method }\end{array}$ & $\begin{array}{c}\text { Fixed power } \\
\text { sharing }\end{array}$ & $\begin{array}{c}\text { Proportional power } \\
\text { sharing }\end{array}$ & $\begin{array}{c}\text { Priority power } \\
\text { sharing }\end{array}$ \\
\hline Droop & No & No & Yes \\
Ratio & No & Yes & No \\
Priority & No & No & Yes \\
$\begin{array}{c}\text { Voltage } \\
\text { margin }\end{array}$ & Yes & Yes & Yes \\
\hline
\end{tabular}

According to the comparisons of Tables 1 and 2, voltage margin method has desirable features, including high expandability and flexibility and also it has the potential of implementing all three schemes of power sharing, which cause in this paper, this method is used to control DC voltage control. Following, this method will be investigated.

\subsubsection{Voltage Margin Control}

The main strategy of voltage margin control method includes a DC voltage controller with a limiter in its output. DC voltage controller uses a PI controller. Limiter is used to restrict reference amount of d-axis current to an upper and a lower amount, in order to avoid overcurrent in converter valves. Therefore, converter active power is also restricted to an upper and lower amount. Thus, DC voltage controller, while active power flows in the converter and is between predefined limits, will be able to keep DC voltage equal to the reference amount [21]. Lower limit and upper limit of reference amount of d-axis current obtain through (7) and (8), respectively [21]:

$$
\begin{aligned}
i_{d, \text { lower }}^{*} & =\frac{p_{\text {lower }}-e_{q} i_{q}^{*}}{e_{d}} \\
i_{d, \text { upper }}^{*} & =\frac{p_{\text {upper }}-e_{q} i_{q}^{*}}{e_{d}}
\end{aligned}
$$

Where $\mathrm{i}_{\mathrm{q}}^{*}$ is the reference amount of $\mathrm{q}$-axis current, which is created by reactive channel outer controllers; $\mathrm{e}_{\mathrm{q}}$ and $\mathrm{e}_{\mathrm{d}}$ are PCC voltages in $\mathrm{d}-\mathrm{q}$ frame; and $\mathrm{p}_{\text {lower }}$ and $\mathrm{p}_{\text {upper }}$ are inverter and rectifier limits, respectively. Voltage margin method based on power sharing plan, in converters as single-stage or two-stage. As stated, voltage margin method is able to implement all three schemes of fixed, proportional, and priority power sharing [22]. In this paper, in order to implement voltage margin method, fixed power sharing scheme has been considered. DC voltage controllers will implement only in grid side converters. Considering that test grid has two grid side converters, in the first converter, two-stage DC voltage controller, and in the second converter, single-stage DC voltage controller are implemented.

\section{HBMO Algorithm}

In this paper, HBMO algorithm is used to optimize parameters of each VSC controller. HBMO algorithm at first proposed by Abbass in 2001 [22]. This algorithm has been successfully applied to solve job planning, data mining, nonlinear constrained and unconstrained optimization, stochastic dynamic programming, and continuous optimization problems [23]. 
HBMO algorithm can be summarized in five basic steps [22]:

1. The algorithm starts with mating flight, in which a queen (best solution) selects drones probabilistically to fill her sperm chamber, and finally creation of broods.

2. New broods (trial solutions) are created by crossover ring drones' genotypes with the queen's.

3. Workers (heuristics) are used to conduct local search (breeding and promotion of broods).

4. Workers' fitness is adapted based on the degree of improvement achieved on broods.

5. In this process, the fitter brood, in the case of superiority over the existing queen, is selected for replacement of queen and doing the next mating flight.

In order to optimize controllers, it is necessary to define a fitness function or a performance index. In this study, according to [16], performance index $I$ is defined based on minimization time integral, multiply by absolute error, which is presented in (9):

$$
\text { MinimizeI }=\int_{0}^{T} t\left|h_{r}-h_{m}\right| d t
$$

Where $T$ is optimization time period and $h_{r}$ and $h_{m}$ are reference and measured amounts of output. HBMO algorithm tries to minimize performance index I during simulation, in offline mode. Then, optimal controllers are used for MTDC grid control.

\section{Tuning of Controllers}

As mentioned previously, in order to investigate performance of optimal controllers, a four-terminal DC grid including two offshore wind farms and two onshore AC grids is used. In Figure 2, schematic diagram of this grid is shown. It should be noted that in offshore converters, ICC and also active power controllers are used as outer controllers and for AC grid converters, ICC and DC voltage controller are applied. Therefore, given that ICC is the same for all four terminals; it is regulated only for one station. Moreover, active power controller is also regulated for one offshore station and DC voltage controller is also set for one onshore

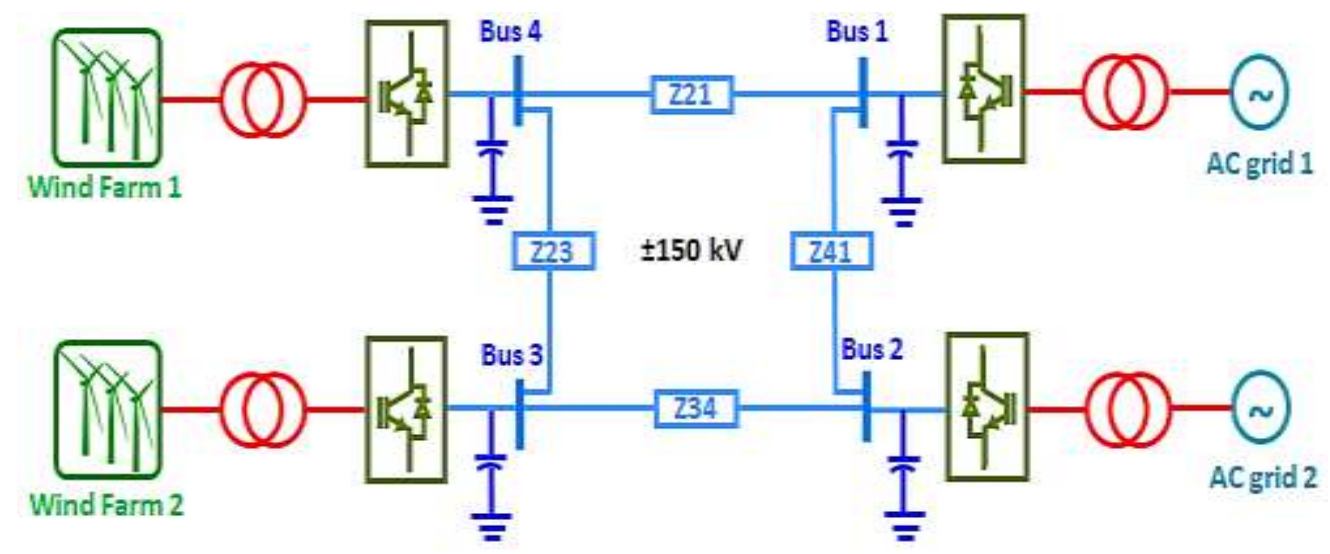

Figure 2. Four-terminal DC Grid including Two Onshore AC Grids and Two Offshore Wind Farms [16] 


\subsection{Tuning of ICC}

In order to optimize ICC, a step signal is applied in d-axis as input reference current for ICC, and PI controller parameters, i.e., $K_{I, P}$ and $K_{I, I}$, are optimized through implementing HBMO algorithm. Since PI controllers in ICC are the same for d-axis and q-axis, step input is only used for d-axis current, while a zero input is considered for q-axis. According to the defined performance index in (9), for optimizing ICC, performance index is stated as (10) [16]:

$$
\text { Minimize I ICC }_{I}=\int_{0}^{T} t\left|i_{d}-i_{d, r e f}\right| d t
$$

\subsection{Tuning of Active Power Controller}

Here, active power controller is used as outer controller in offshore VCSs. In order to set active power controller, at first optimized ICC is used in VSC control structure, and then active power controller is optimized by the aid of HBMO algorithm. In order to optimize active power controller such as ICC optimization, a step signal is applied to active power controller as power input reference, and PI controller parameters, i.e., $K_{P, P}$ and $K_{P, I}$ are optimized.

In fact, the aim of regulation power controller is optimization of PI parameters, such that measured active power in VSC follows reference value (step signal). For optimizing active power controller, performance index $I$ is obtained from (11) [16]:

$$
\text { Minimize }_{P C}=\int_{0}^{T} t\left|p-p_{\text {ref }}\right| d t
$$

\subsection{Tuning of DC Voltage Controller}

If voltage margin method is used as DC voltage controller in grid side converters, PI parameters of voltage margin control are set such as active power controller in offshore converters. So that at first ICC should be set and DC voltage controller should create d-axis reference value for optimal ICC.

Given that the nature of DC voltage controller is the same in both converters of AC grid side, voltage controller can be set for one converter and then the other converter be set similarly. Like optimizing other controllers, for optimal regulation of DC voltage controller, a step signal is applied to controller as a reference value, and it is trying to optimize PI gains, i.e., $K_{V, P}$ and $K_{V, I}$, such that DC voltage follows its reference value. Therefore, performance index $I$ is defined for DC voltage controller as (12):

$$
\text { Minimize }_{D C}=\int_{0}^{T} t\left|V_{D C}-V_{D C, r e f}\right| d t
$$

\section{Simulation Results}

In simulation section, at first inner current controller and outer controllers are set classically, and then optimal parameters of these controllers are obtained by the aid of HBMO algorithm, and the results of optimal setting and classic regulation are compared with each other.

\subsection{Classic Tuning of ICC and Outer Controllers}

PI controller parameters are set according to the following points, classically [24]:

- To achieve a desirable performance, ICC closed-loop bandwidth shouldn't be more than 1.5 switching angular frequency. 
- For response of closed- loop system be not oscillatory, outer controllers should be considered at least 10 times slower than ICC.

According to the above points, PI controller parameters for ICC, are set as (13) and (14) $[21]:$

$$
\begin{aligned}
& K_{I, P}=\alpha_{c} L_{T}^{p u} \\
& K_{I, I}=\alpha_{c} R_{T}^{p u}
\end{aligned}
$$

Where, $\alpha_{c}$ is ICC closed-loop bandwidth.

Since in this study, switching frequency is set less than $2 \mathrm{KHz}$, so:

$$
\alpha_{c}<\frac{2 \times \pi \times 2}{5}=2.5 \mathrm{Krad} / \mathrm{s}
$$

According to safe margin, the value of $2 \mathrm{Krad} / \mathrm{s}$ is considered for $\alpha_{c}$.

Also, in order to set outer controllers parameters, this issue should be considered that for closed-loop system response not be oscillatory, it is required that these controllers be at least 10 times slower than ICC. So, width of outer controllers' bandwidth can be obtained from (16):

$$
\alpha_{o c}=0.1 \alpha_{c}
$$

According to [21], integral and proportional gains of outer controllers can be obtained from (17) and (18):

$$
\begin{aligned}
K_{O, P} & =\alpha_{o c} C^{p u} \\
K_{O, I} & =\alpha_{o c}^{2} C^{p u}
\end{aligned}
$$

\subsection{Optimal Tuning of ICC and Outer Controllers}

In this section, controllers' parameters for a VSC station are optimized by HBMO algorithm. Parameters of HBMO algorithm are presented in Table 3.

Table 3. Parameters of HBMO Algorithm

\begin{tabular}{lccc} 
Parameter & $\begin{array}{l}\text { Number of } \\
\text { variables }\end{array}$ & $\begin{array}{l}\text { Initial population of } \\
\text { drones }\end{array}$ & $\begin{array}{l}\text { Number of mating } \\
\text { flights }\end{array}$ \\
\hline Value & 2 & 30 & 15 \\
\hline
\end{tabular}

By applying HBMO algorithm in ICC and outer controllers (active power and DC voltage), optimal algorithm performance in controllers is observed. Figure 3 shows the value of performance index during optimizing ICC controllers and outer controllers, by HBMO algorithm. 


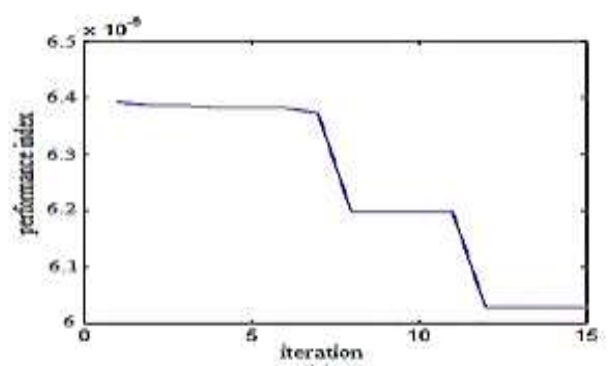

(a)

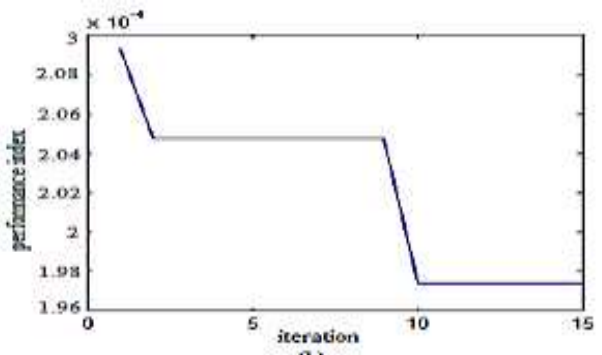

(b)

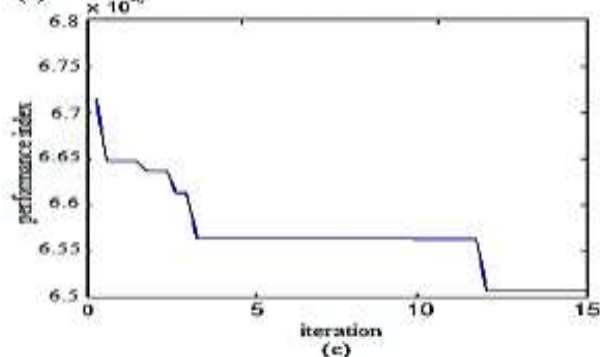

Figure 3. Performance Index during Optimizing, (a) ICC, (b) Active Power Controller (c), DC Voltage Controller

Resulting parameters from optimizing ICC and outer controllers obtained from classic setting and optimal setting are presented in Table 4 .

Table 4. Resulting Parameters from Optimal and Classic Tuning of ICC and Outer Controllers

\begin{tabular}{ccccc}
\hline Controller & \multicolumn{2}{c}{ Classic setting } & \multicolumn{2}{c}{ HBMO algorithm } \\
& $\mathrm{K}_{\mathrm{p}}$ & $\mathrm{K}_{\mathrm{i}}$ & $\mathrm{K}_{\mathrm{p}}$ & $\mathrm{K}_{\mathrm{i}}$ \\
\hline ICC & 0.95 & 10 & 1.96 & 29.51 \\
$\begin{array}{c}\text { Active power } \\
\text { controller }\end{array}$ & 0.54 & 81 & 0.19 & 99.62 \\
$\begin{array}{c}\text { DC voltage } \\
\text { controller }\end{array}$ & 0.54 & 81 & 3.83 & 303.03 \\
\hline
\end{tabular}

After optimizing controllers, performance of control system is examined for a VSC station; such that response of each controller to the change of step input is observed. In order to compare optimal and classic setting modes, step response of controllers is presented in a diagram.

In Figure 4 (a), step response of ICC in classic and optimal setting modes optimized by HBMO algorithm for d-axis current is observed. It is clear that ICC optimized by HBMO algorithm has better performance than classic setting mode. Considering that current in $\mathrm{d}$-axis reached value 1 ( $d$-axis reference input) faster and followed it better, performance of ICC optimized by HBMO algorithm.

Performance of active power controller in classic setting mode and optimized by HBMO algorithm, when a step reference value is applied to the controller, is shown in Figure 4 (b). From this figure we can conclude that active power controller optimized by HBMO algorithm, comparing setting it classically, has faster reaction to the reference power of step input and has followed reference input better. Thus performance of active power controller is optimized by using HBMO algorithm. 
Also, in order to compare classic setting of voltage margin controller with optimal setting, its parameters by HBMO algorithm, step response of voltage margin controller are presented in Figure 4(c).

It is clear that optimized voltage margin controller by HBMO algorithm has better performance than classic regulation of this controller. It is seen that DC voltage regulated by classic method, at first had oscillatory response and subsequently couldn't follow its reference value, well; but diagram of DC voltage optimized by HBMO algorithm has reached its reference value without having oscillation and very fast, and could follow it very well. Thus performance of voltage margin controller optimized by using HBMO algorithm.

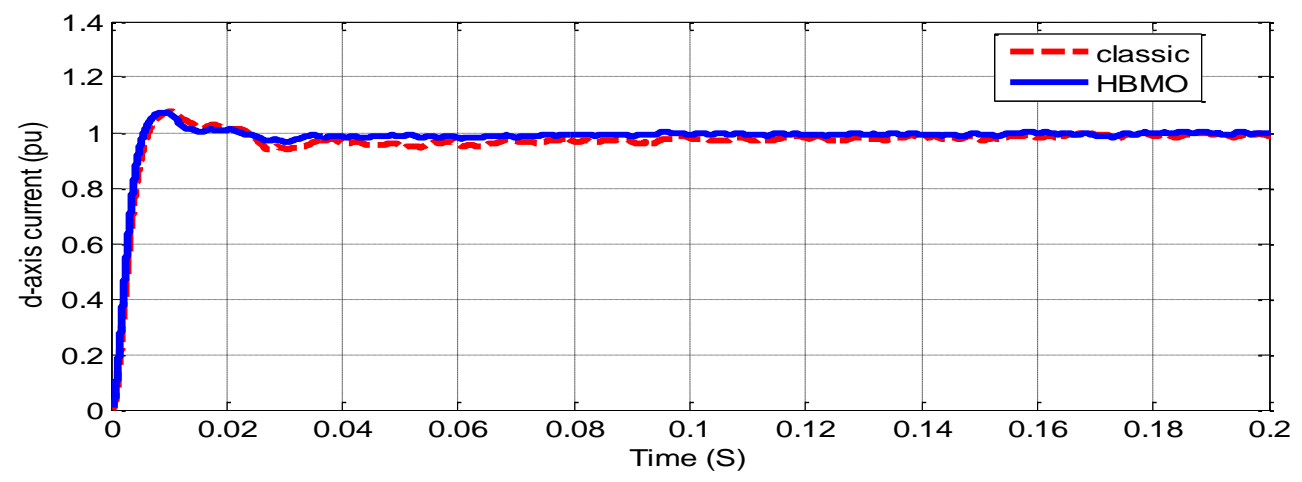

(a)

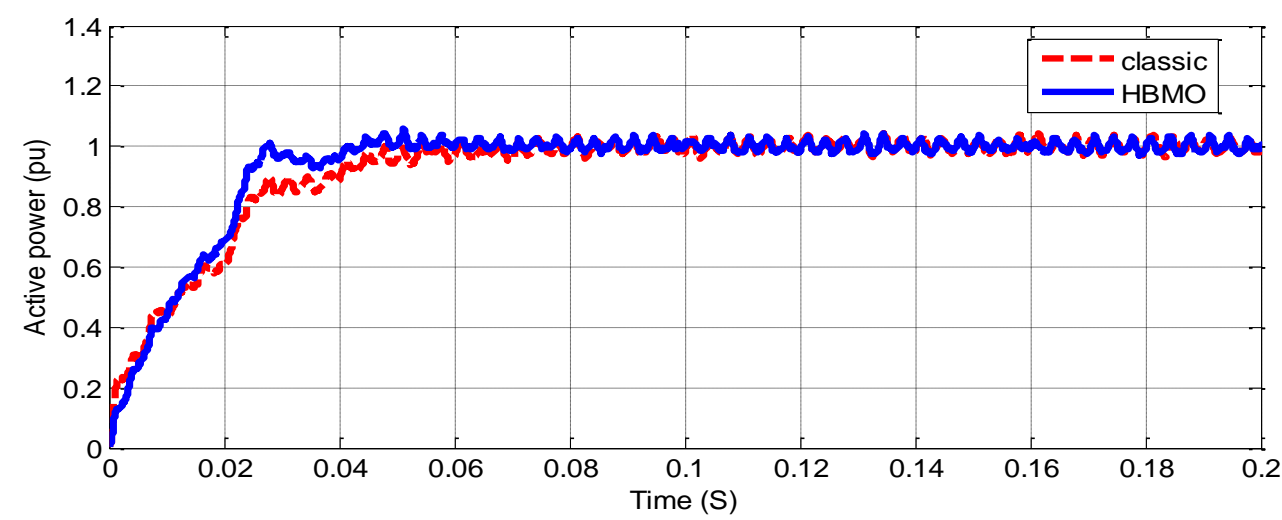

(b)

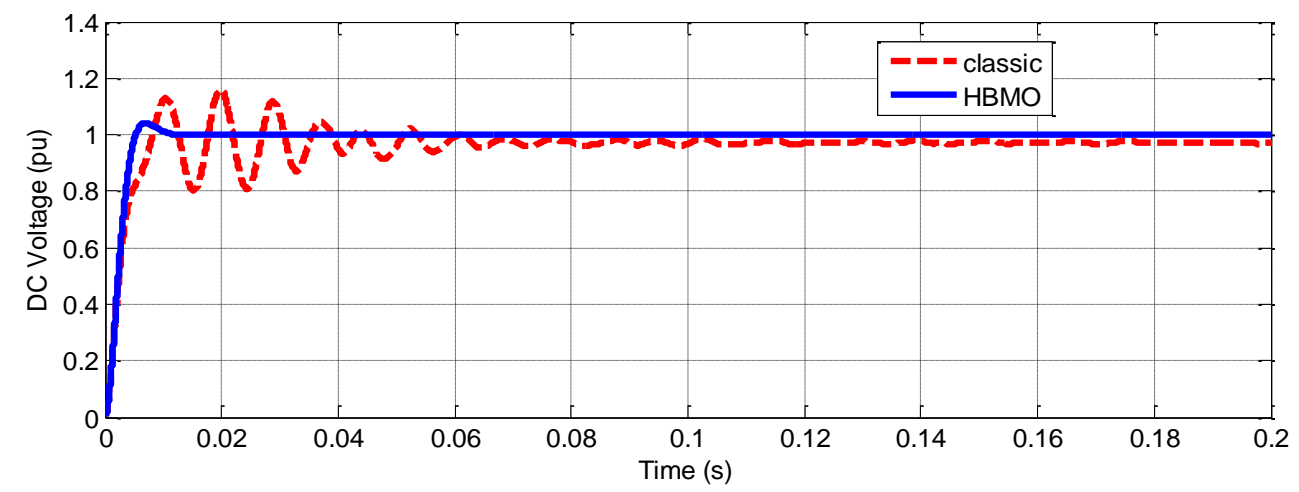

(c)

Figure 4. Step Response in Classic Setting Mode and Optimal Setting Mode for, (a) ICC, (b) Active Power Controller, (c) Voltage Margin Controller 
Numerical comparison of performance of ICC and outer controllers in classic setting mode and optimized by HBMO algorithm, in terms of performance defined in (9), is presented in Table 5.

Table 5. Comparison between MTDC Grid Controllers in Classic Tuning and Optimal Tuning Mode in Terms of Performance Index

\begin{tabular}{lll}
\hline Controller & $\begin{array}{l}\text { Performance index } \\
\text { Classic setting }\end{array}$ & HBMO algorithm \\
\hline ICC & $3.08 \times 10^{-4}$ & $6.03 \times 10^{-5}$ \\
active power controller & $1.33 \times 10^{-4}$ & $1.97 \times 10^{-4}$ \\
DC voltage controller & $2.79 \times 10^{-4}$ & $6.52 \times 10^{-6}$ \\
\hline
\end{tabular}

Given that performance index for optimizing VSC controllers is defined based on minimizing them, according to the above table it is seen that the results of HBMO algorithm for ICC and outer controllers are optimized under identical conditions than obtained results from setting these controllers classically.

\subsection{Investigating Performance of Controllers Optimized by HBMO Algorithm in MTDC Grid}

In order to study performance of offered controllers in MTDC grid, a four-terminal test grid is used, as it is shown in Figure 2. Test system is composed of two AC grids (onshore) with nominal voltage level $220 \mathrm{kV}$ and rated power $2 \mathrm{GW}$ and two offshore wind farms with nominal voltage $33 \mathrm{kV}$ and capacity of $150 \mathrm{MW}$. Nominal voltage of DC grid is $\pm 150 \mathrm{kV}$.

Parameters of four-terminal DC grid are presented in table 6. Complete model of four-terminal DC test grid is used for conducting simulation and confirming performance of suggested controllers.

Table 5. Parameters of Four-terminal Test Grid [16]

\begin{tabular}{clll}
\hline Model & $\begin{array}{l}\text { Rated voltage } \\
(\mathrm{kV})\end{array}$ & Impedance & Descriptions \\
\hline $\begin{array}{c}\text { AC } \\
\text { networks }\end{array}$ & 220 & $0.001+\mathrm{j} 0.15 \mathrm{pu}$ & $2000 \mathrm{MVA}$ \\
$\begin{array}{c}\text { Wind } \\
\text { farms }\end{array}$ & 33 & $0.002+\mathrm{j} 0.1 \mathrm{pu}$ & $150 \mathrm{MVA}$ \\
$\begin{array}{c}\text { Phase } \\
\text { reactor }\end{array}$ & 180 & $0.01+0.15 \mathrm{pu}$ & - \\
$\begin{array}{c}\text { DC } \\
\text { capacitor }\end{array}$ & \pm 150 & $\begin{array}{l}\text { Two } 100 \mu \mathrm{F} \text { capacitors } \\
\text { connected in series }\end{array}$ & - \\
& & $\mathrm{R}=1.13 \times 10^{-2} \Omega / \mathrm{km}$ & \\
DC link & \pm 150 & $\mathrm{~L}=1.59 \times 10^{-4} \mathrm{H} / \mathrm{km}$ & $\begin{array}{l}100 \mathrm{~km} \text { between all } \\
\text { stations }\end{array}$ \\
\hline
\end{tabular}

Two AC grid side converters in DC four-terminal grid use voltage margin method as DC voltage controllers. Desirable performance of these controllers should be such that power balance in the grid is maintained, consequently in this regard, voltage 
margin method uses fixed power sharing scheme. In order to implement fixed power sharing scheme, VDC-P characteristics of Fig. 5 has been used for two AC grid side converters.

It should be mentioned that reactive channel in grid side converters, act like reactive power control and tries to control power factor through neutralizing exchanged reactive power in $\mathrm{AC}$ grids at unit value.

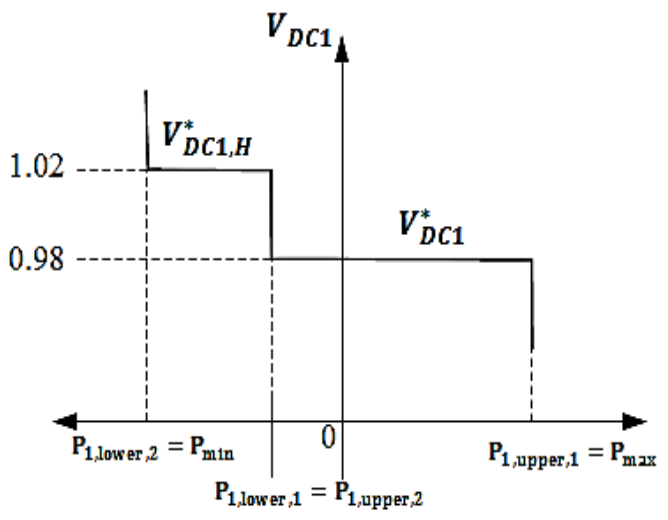

(a)

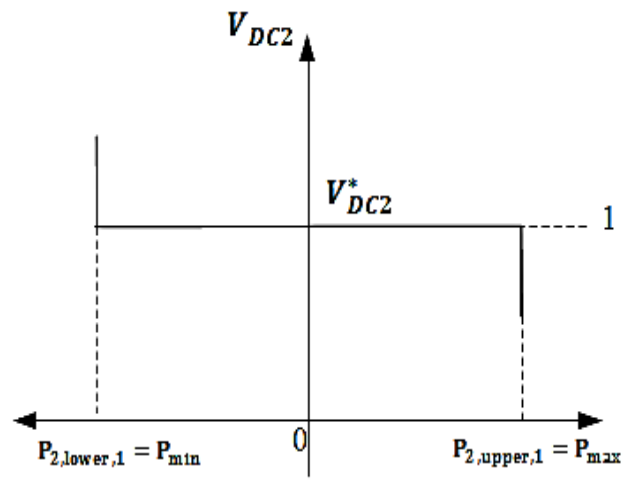

(b)

Figure 5. Characteristics of VDC-P, (a) AC1 Converter, (b) AC2 Converter [21]

Here, values of $P_{\max }$ and $P_{\min }$ are considered 3 and -3 , respectively. Also, the first converter receives a certain amount of active power, $p_{1, \text { lower }, 1}=0.4 p u$, which maybe this amount is generated in offshore wind farms, or transferred from another onshore converter, or a combination of these two. On the other hand, the second converter is responsible of creating power balance in MTDC grid.

At first, considering that the first converter has less reference value, it controls DC voltage and then the second converter controls DC voltage, and finally, when DC voltage is more than the reference value of the second converter, the first converter begins to control DC voltage in the second step.

In order to investigate performance of optimal controllers in a MTDC grid, the proposed scenario in table 7 is used for generating power in offshore converters. The proposed scenario includes step changes in output power of wind farms.

Table 5. Scenario of Power Generation in Two Offshore Wind Farms of Four-terminal DC Grid [16]

\begin{tabular}{llll}
\hline Event time & $\mathrm{t}=0 \mathrm{sec}$ & $\mathrm{t}=0.5 \mathrm{sec}$ & $\mathrm{t}=0.8 \mathrm{sec}$ \\
\hline wind farm 1 power pu & 0.4 & 0.4 & 0.8 \\
wind farm 2 power pu & 0.8 & 1.2 & 1.2 \\
\hline
\end{tabular}

At the beginning of simulation, wind farms 1 and 2 generate $0.4 \mathrm{pu}$ and $0.8 \mathrm{pu}$ power, respectively. This situation continues until $\mathrm{t}=0.5 \mathrm{sec}$. At this moment, generated power by wind farm 2 increases up to $1.2 \mathrm{pu}$. Finally, at the $\mathrm{t}=0.8 \mathrm{sec}$, generated power by wind farm 1 increases up to 0.8 pu.

Appropriate performance of optimal controllers depends on this issue that AC grid side converters follow changes of generated power by wind farms and consequently maintain power balance in the grid.

Since at the beginning moment of simulation, wind farm 1 and wind farm 2 generate $0.4 \mathrm{pu}$ and $0.8 \mathrm{pu}$, respectively, in the very beginning the first converter receives $0.4 \mathrm{pu}$ 
fixed power and the second converter should also maintain power balance within whole grid. Therefore, the second station complies with power change in wind farms. As it is shown in Figure 6 , at moment $t=0.5 \mathrm{sec}$, generated power by wind farm 2 increases up to $1.2 \mathrm{pu}$, as a result, power of the second converter increases in inversion mode, such that power balance is maintained within the grid, and finally by increasing generated power by wind farm 1 at the moment $\mathrm{t}=0.8 \mathrm{sec}$ up to $0.8 \mathrm{pu}$, as expected, power of the second converter is also increases in inversion mode. Therefore, power balance in the grid has been maintained well and optimized DC voltage controller has presented desirable performance.

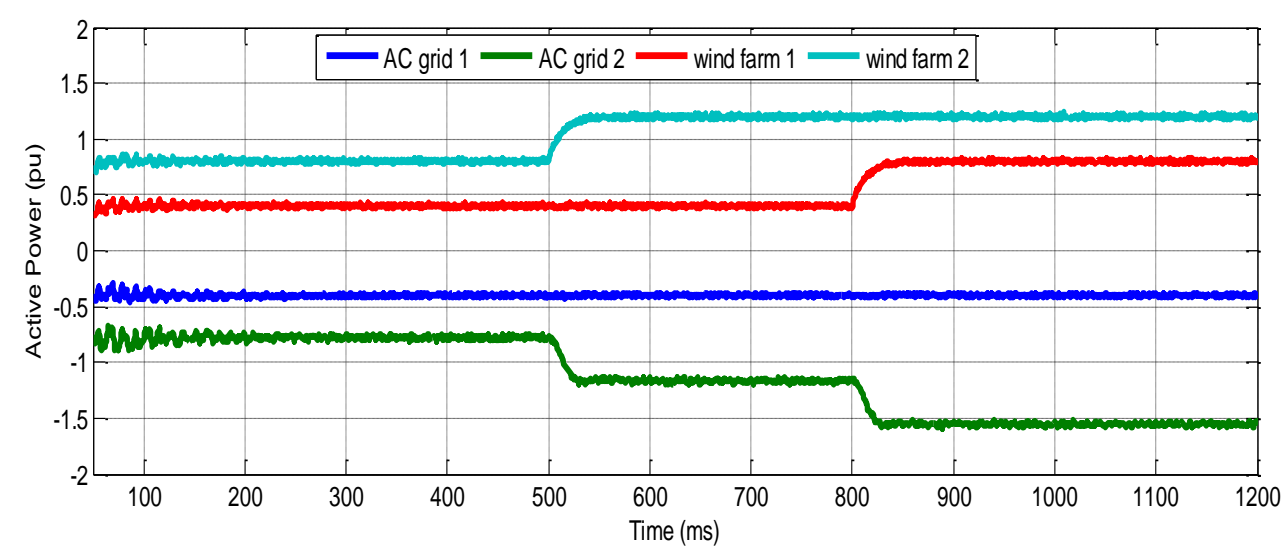

Figure 6. Active Power of Four-terminal DC Grid Converters Optimized by HBMO Algorithm

In Figure 7, diagrams of voltage in DC terminals of MTDC grid are also presented. As it is observed, with any change of power in wind converters, a peak moment is applied to DC voltage, and again DC voltage is placed around its setting point.

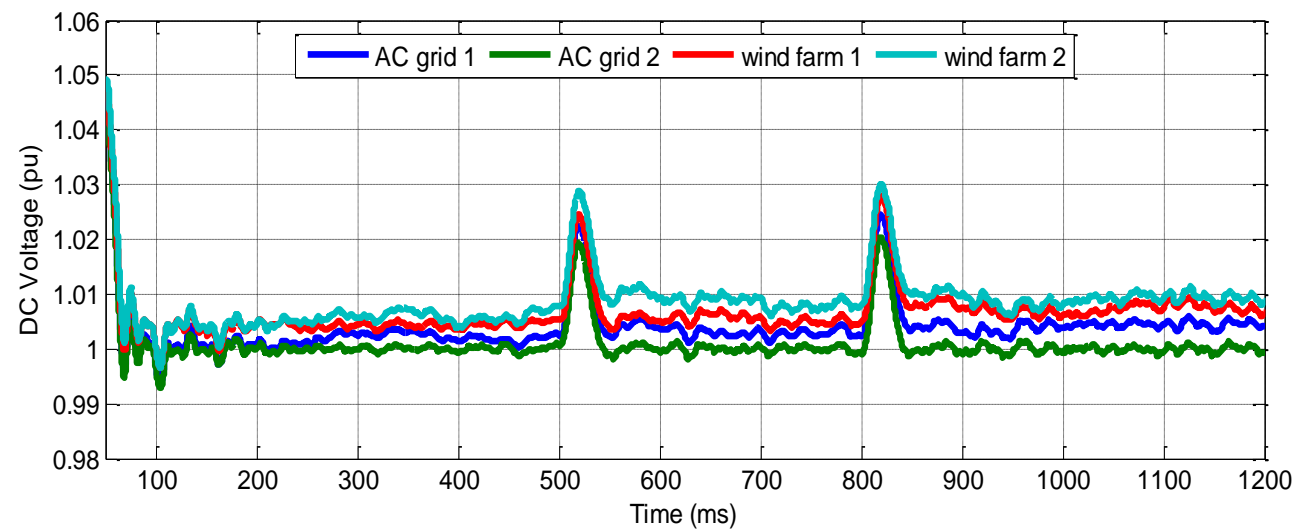

Figure 7. DC Voltage of Four Terminal DC Grid Converters Optimized by HBMO Algorithm

Now, in order to compare performance of MTDC grid in classic regulation mode and optimal regulation by HBMO algorithm, in the case of using voltage margin method as DC voltage controller, Figure 8 is presented that shows DC voltage diagram in station AC1. As it is observed, voltage peak in optimal regulation mode by HBMO algorithm has almost been halved comparing with classic mode, and also voltage in optimal mode returns to its initial mode faster. Therefore, optimal HBMO algorithm has better performance. 


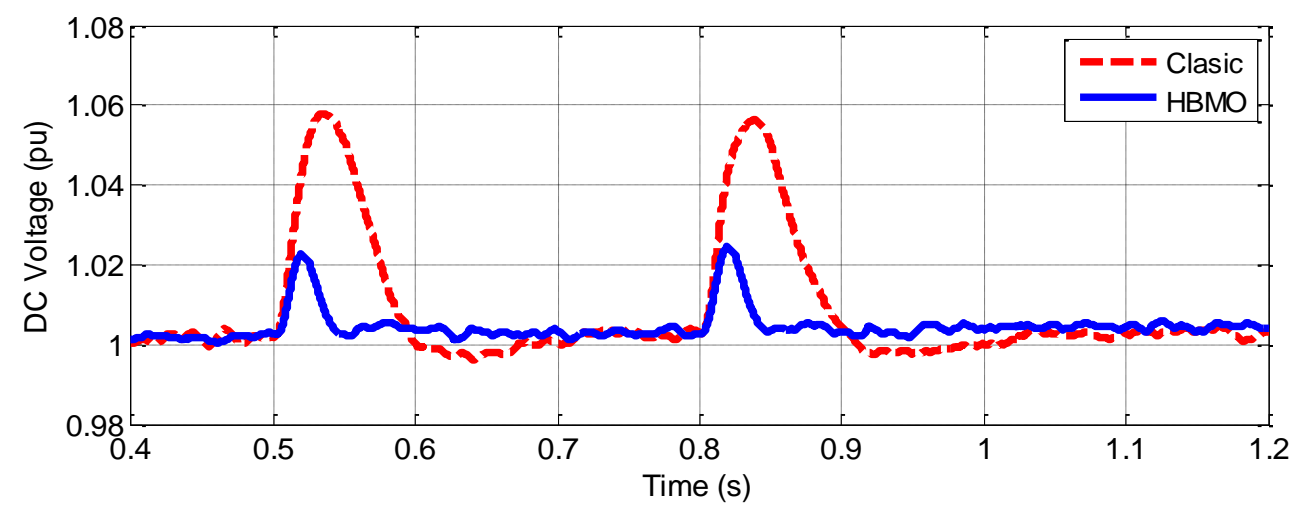

Figure 8. Comparing Classic Setting and Optimal Setting for AC1 Converter DC Voltage of Four Terminal DC Grid

\section{Conclusion and Recommendations}

The main purpose of this research is improving control function of MTDC grid by intelligent optimization algorithms. For this aim, HBMO algorithm is used for optimization. Vector control method is considered as the control method for VSCHVDC grids. ICC and outer controllers in vector control method were optimized by HBMO algorithm. Also voltage margin control method used as a DC voltage controller and advantages of this method relative to other DC voltage control methods were stated. Controllers' parameters, including voltage margin control were optimized by the aid of HBMO algorithm, and the results were compared with classic setting mode and improving performance of optimal controllers relative to classic mode was observed. Then, desirable performance of optimized controllers in a four terminal test grid was observed, and finally a comparison between optimized controllers and classic ones was done in MTDC grid, which they represent desirable control performance of HBMO algorithm comparing with classic setting mode.

\subsection{Recommendations}

In this section, researches that can be conducted in the field of MTDC grids in future are stated:

- Analysis of DC error for MTDC grids can be studied and developed.

- More study of reactive power controllers and AC voltage in vector control method and their optimization.

- Optimizing DC voltage margin method by using priority and relative power sharing schemes.

\section{References}

[1] R. Teixeira Pinto and P. Baure, "The role of modularity inside the north sea transnational grid project: modular concepts for construction and operation of large offshore grids", presented at The Renewable Energy World Europe Conference, Milan, Italy, (2011).

[2] EWEA, "Wind in power 2010 European statistics", Brussels, (2011).

[3] OTEG-GBSQSS, "OTEG offshore Transmission Expert Group-GBSQSS Great Britain Security and Quality of supply sub-group, Recommendations for the coverage of offshore transmission networks in the great Britain Security and Quality of supply standard", (2006).

[4] L. Livermore, J. Liange and K. Ekanayake, "MTDC VSC technology and its application for wind power", 45th International Universities Power Engineering Conference (UPEC), Cardiff, UK, (2010).

[5] M. Saeedifard, M. Graovac and R. F. Dias, "DC power systems: challenges and opportunities", IEEE Power and Energy Society General Meeting, (2010). 
[6] N. Flourentzou, V. Agelidis and G. Demetriades, "VSC-based HVDC power transmission systems: An overview", IEEE Transactions on Power Electronics, vol. 24, no. 3, (2009), pp. 592-602.

[7] M. P. Bahrman and K. Johnson, "The ABCs of HVDC transmission technologies", IEEE Power Energy Mag, vol. 5, no. 2, (2007), pp. 32-44.

[8] E. Veilleux and B. Ooi, "Multi-terminal HVDC with thyristor power-flow controller", IEEE Transaction on Power Delivery, vol. 27, no. 3, (2012), pp. 1205-1212.

[9] R. S. Whitehouse, "Technical challenges of realizing multi-terminal networking with VSC", 14th European Conference On Power Electronics and Applications (EPE), (2011).

[10] X. Chen, H. Sun and J. Wen, "Integrating wind farm to the grid using hybrid multi terminal HVDC technology", IEEE Transactions on Industry Applications, vol. 47, no. 2, (2011), pp. 965-972.

[11] C. Dierckxsens, K. Sirvastava and M. Reza, "A distributed DC voltage control method for VSC-MTDC systems”, Electric Power Systems Research, vol. 82, no. 1, (2012), pp. 54-58.

[12] L. Harnefors, M. Bongiono and S. Lundberg, "Input-Admittance calculation and shaping for controlled voltage source converters", IEEE Trans. Indust. Electron, vol. 54, no. 6, (2007), pp. 3323-3334.

[13] A. Miranian and M. Zarif, "A detailed review on MTDC grids- technical issues and challenges", paperback, LAP LAMBERT Academic Publishing, (2014).

[14] Z. L. Gaing, "Apaticle swarm optimization approach for optimum design of PID controller in AVR system", IEEE Transactions on Energy Conversion, vol. 19, no. 2, (2004), pp. 384-391.

[15] T. L. Seng, M. B. Khalid and R. Yusof, "Tuning of a neuro-fuzzy controller by genetic algorithm”, IEEE Trans. Syst., (1999), pp. 226-236.

[16] K. Rouzbehi, A. Miranian and A. Luna, "Optimized control of multi-terminal DC grids using particle swarm optimization”, Power Electronics and Applications (EPE), Lille, (2013), pp. 1-9.

[17] K. Rouzbehi, A. Miranian and A. Luna, "Optimized control of multi-terminal DC grids using particle swarm optimization", EPE Journal, vol. 24, no. 2, (2014), pp. 38-49.

[18] O. Bozorg Haddad, A. Afshar and M. A. Marino, "Honey-bees mating optimization (HBMO) algorithm: A new heuristic approach for water resources optimization", Water Resources Management, vol. 20, no. 5, (2006), pp. 661-680.

[19] L. Zhang, Y. Wang and H. Li, "Coordinated control of MTDC-based micro grid with wind turbines", in 7th International Power Electronics and Motion Control Conference (IPEMC), (2012).

[20] N. R. Chaudhuri and B. Chaudhuri, "Adaptive droop control for effective power sharing in multi-terminal DC (MTDC) grids”, IEEE Trans. Power syst., vol. 28, no. 1, (2013), pp. 21-29.

[21] S. Rodrigues, "Dynamic modeling and control of VSC-based, Multi-terminal DC networks", M.Sc. thesis, Universidade Tecnica de Lisboa, (2011).

[22] H. A. Abbass, "Marriage in Honey Bees Optimization (MBO): A Haplometrosis Polygynous swarming approach", The Congress on Evolutionary Computation, Seoul, Korea, (2001), pp. 207-214.

[23] A. Baykasoùlu, L. Özbakır and P. Tapkan, "Artificial bee colony algorithm and its application to generalized assignment problem", Itech Education and Publishing, Vienna, Austria, (2007), pp. 113-144.

[24] R. T. Pinto, P. Bauer and S. Rodrigues, "A novel distributed direct voltage control strategy for grid integration of offshore wind energy systems through MTDC network", IEEE Transactions on Industrial Electronics, vol. 60, no. 6, (2013) June, pp. 2429-2441. 
International Journal of Control and Automation

Vol. 11, No. 10 (2018) 